\title{
Mediastinal basaloid carcinoma arising from thymic cysts in two dogs
}

\author{
Yuto SANO ${ }^{1) *}$, Kanae SEKI ${ }^{1)}$, Kenjirou MIYOSHI ${ }^{2)}$, Toshikazu SAKAI'), \\ Tsuyoshi KADOSAWA ${ }^{2)}$ and Kazuya MATSUDA ${ }^{1 /}$
1) Department of Veterinary Pathology, School of Veterinary Medicine, Rakuno Gakuen University, Ebetsu, Hokkaido 069-8501, Japan
${ }^{2)}$ Department of Small Animal Clinical Sciences, School of Veterinary Medicine, Rakuno Gakuen University, Ebetsu, Hokkaido 069-8501, Japan

\author{
J. Vet. Med. Sci. \\ 83(5): 876-880, 2021 \\ doi: 10.1292/jvms.20-0518 \\ Received: 1 September 2020 \\ Accepted: 24 March 2021 \\ Advanced Epub:
}

5 April 2021

\begin{abstract}
Mediastinal masses in dogs were diagnosed as basaloid carcinoma associated with multiple thymic cysts (MTCs). The masses were composed of MTCs and proliferating intracystic neoplastic basaloid cells, which immunohistochemically diffusely expressed p63 and cytokeratin 19. A gradual transition from the basal cell layers lining the cysts walls to the neoplastic cells was seen, and it was indicated that the neoplastic cells had originated from the basal cell layers of the cysts. To the best of our knowledge, this is the first report of basaloid carcinoma occurring in the mediastinal cavity in dogs. Although these tumors were demonstrated to be rare origins, basaloid carcinoma should be included in the differential diagnoses for canine mediastinal tumors.
\end{abstract}

KEY WORDS: basaloid carcinoma, dog, mediastinum, thymic branchial cyst, thymic cyst

Basaloid carcinoma is a malignant epithelial tumor. It has similar characteristics to basal cell carcinoma, which affects the epidermis. In the human World Health Organization (WHO) classification, basaloid carcinoma has been classified under tumors of the thymus [12]. There is no definitive tumor classification for basaloid carcinoma in animals, and there has only been one case report about basaloid squamous cell carcinoma (BSCC) occurring in dogs [10].

Cystic lesions can occur in various regions, and they are diagnosed based on their locations and the histological characteristics of their epithelial linings and stroma. Among them, cystic lesions located in the mediastinal cavity that arise in developing, mature, or regressive thymi are called thymic cysts [15]. Although their occurrence in dogs is rare, such canine lesions have previously been reported as thymic branchial cysts $[3,8]$.

Tumors associated with cystic lesions have been reported in the thymus in humans $[2,6,12]$, and among the tumors that arise in the thymus, the formation of cystic lesions occurs sporadically in basaloid carcinoma, suggesting that a relationship exists between cysts and tumor formation. In dogs, there have been two reports of malignant tumors associated with cystic lesions in the thymus $[1,7]$; however, these lesions were not referred to as basaloid carcinoma. In this report, we describe two cases in which mediastinal tumors associated with cystic lesions; i.e., basaloid carcinoma arising from thymic cysts, occurred in dogs.

Case 1 involved an 11-year-old, spayed Chihuahua, and case 2 involved a 9-year-old, castrated Yorkshire terrier. The case 1 was taken to a primary hospital with chief complaints of depression, loss of balance, a lack of appetite, and cough, which had lasted for a few days. The case 2 was with complains of depression and a lack of appetite, and the patient also presented pale tongue mucous membrane and labored breathing. In each case, clinical examinations revealed a mass in the anterior mediastinum. Thus, the dogs were referred to the Department of Veterinary Surgery of Rakuno Gakuen University Animal Medical Center to undergo detailed examinations. In case 1, radiographic examinations demonstrated an indistinct heart shadow, increasing mediastinal opacity, and a mediastinal mass, and dorsal-ventral imaging showed that the mass had compressed the trachea, pushing it in the right lateral direction. On computed tomography (CT), the mediastinal mass, which measured approximately $4.3 \times 3.8 \times 5.7 \mathrm{~cm}$, containing a materials with a slightly low CT value separated by irregularly thickened structure was found (Supplementary Fig. 1a). In case 2 , radiographic examinations showed that the mediastinal mass had compressed the heart, pushing it in the left caudal direction, and it had also compressed the diaphragm and pushed the trachea in the dorsal direction. The presence of the mediastinal mass with heterogeneous CT value was demonstrated on CT, which measured approximately $6.5 \times 4.5 \times 5.7 \mathrm{~cm}$ in size (Supplementary Fig. 1b). Ultrasound examinations exhibited that the mass had a poor blood supply, and the contents of the mass were homogenous. No metastasis to other organs was observed on CT in either case. To ameliorate the dog's symptoms, the masses were removed via

*Correspondence to: Sano, Y.: y-sano@rakuno.ac.jp

(Supplementary material: refer to PMC https://www.ncbi.nlm.nih.gov/pmc/journals/2350/)

(O2021 The Japanese Society of Veterinary Science

This is an open-access article distributed under the terms of the Creative Commons Attribution Non-Commercial No Derivatives (by-nc-nd) License. (CC-BY-NC-ND 4.0: https://creativecommons.org/licenses/by-nc-nd/4.0/) 
Table 1. Primary antibody used for the immunohistochemical examination in two mediastinal tumors, normal canine skin, liver, and adrenal tissues

\begin{tabular}{lllccl}
\hline \multicolumn{1}{c}{ Antibody } & \multicolumn{1}{c}{ Clone } & Type & Antigen retrieval & Dilution & \multicolumn{1}{c}{ Source } \\
\hline Pan-CK & AE1/AE3 & MM & AC & Prediluted & Nichirei, Tokyo, Japan \\
p63 & 4A4 & MM & MW & $1: 50$ & Thermo Fisher Scientific, Waltham, CA, USA \\
Ki-67 & MIB-1 & MM & MW & $1: 50$ & Dako, Glostrup, Denmark \\
CK5/6 & CK5/6.007 & MM & MM & $1: 50$ & Biocare Medical, Pacheco, CA, USA \\
CK7 & RCK105 & MM & MW & $1: 50$ & Abcam, Cambridge, UK \\
CK8 & Ks8.7 & MM & MW & $1: 50$ & Progen, Heidelberg, Germany \\
CK14 & LL002 & MM & MW & $1: 50$ & Bio-Rad, Hercules, CA, USA \\
CK18 & Ks18.04 & MM & MW & $1: 20$ & Progen \\
CK19 & BA17 & MM & MW & $1: 50$ & Thermo Fisher Scientific \\
$\alpha-S M A$ & 1A4 & MM & MW & $1: 100$ & Dako \\
Syna & SY38 & MM & MW & $1: 30$ & Abcam \\
NSE & BBS/NC/VI-H14 & MM & - & Prediluted & Dako \\
Ch A & - & RP & MW & $1: 300$ & Abcam \\
\hline
\end{tabular}

CK: cytokeratin, SMA: smooth muscle actin, Syna: synaptophysin, NSE: neuron specific enolase, Ch A: chromogranin A, MM: mouse monoclonal, RP: rabbit polyclonal, AC: autoclave, MW: microwave.

median sternotomy. The extracted mediastinal masses were submitted for histopathological examinations.

The tissue samples were fixed in 10\% neutral-buffered formalin, processed routinely, and embedded in paraffin wax. Sections $(4 \mu \mathrm{m})$ were stained with hematoxylin and eosin or periodic acid Schiff, before being subjected to light microscopic examinations. In addition, immunohistochemical examinations were carried out using the immunoenzyme polymer method (Histofine Simple Stain MAX-PO; Nichirei, Tokyo, Japan). Primary antibodies against the following molecules were used for the examinations: pan-cytokeratin (CK), p63, Ki-67, CK5/6, CK7, CK8, CK14, CK18, CK19, $\alpha$-smooth muscle actin (SMA), synaptophysin (Syna), neuron-specific enolase (NSE), and chromogranin A (Ch A). The details of the antibodies used are summarized in Table 1. In each immunohistochemical examination, canine skin tissue was used as a positive control for the detection of pan-CK, p63, Ki-67, CK5/6, CK14, and $\alpha$-SMA, and liver tissue was used as a positive control for the detection of CK7, CK8, CK18, and CK19. For Syna, NSE, and Ch A, the adrenal gland was used as a positive control.

Histologically, the mediastinal masses consisted of multiple non-neoplastic cysts of varying size containing granular eosinophilic materials and a large cyst, which contained proliferating intracystic neoplastic tissue (Fig. 1). The cyst walls were comprised of one or more layers of epithelial cells and were surrounded by fibrous tissue of varying thickness (Fig. 2a). Thymic tissue was frequently found adjacent to the cyst walls (Fig. 2b). The innermost epithelial cells of the cysts included ciliated or unciliated columnar to cuboidal cells and unciliated squamous cells (Fig. 2c), and transitions to epithelial cells with different morphologies were occasionally seen. In case 1, the wall of the large cyst with neoplastic tissue contained thick fibrous tissue. Several islands of proliferating neoplastic cells extended contiguously from the fibrous tissue of the inner wall to the intracystic cavity and exhibited papillary growth. Necrotic tissue, and eosinophilic material, and partially formed cholesterol crystals accumulated in the central region of the cavity. On the other hand, the part of the cyst wall containing neoplastic tissue was composed of thin pieces of fibrous tissue in case 2, and proliferating neoplastic cells filled most of the intracystic cavity. The neoplastic tissues of the two cases were composed of papillary and/or diffuse stratified proliferating neoplastic cells accompanied by scant to fine fibrovascular stroma. The neoplastic cells had proliferated, producing cystic spaces lined by multiple layers of tumor cells, which were filled with eosinophilic material or red blood cells (Fig. 3a). In the peripheral regions of neoplastic cell proliferation, the nuclei of the cells often demonstrated palisading along the interface with the stroma (Fig. 3b). Mild tumor cell invasion was seen in the adjacent cyst wall (Supplementary Fig. 2a and 2b). The neoplastic cells had relatively round to oval nuclei and were medium size. They exhibited a pale to granular chromatin pattern, small distinct nucleoli, moderate amounts of eosinophilic cytoplasm, and indistinct cell borders. The surface of the neoplastic tissue was occasionally covered with an epithelial layer. In the peripheral region of the neoplastic tissue, continuity with the innermost epithelial cells lining the cystic wall was noted. In addition, a gradual transition from the basal cell layer to the islands of proliferating neoplastic cells was evident (Fig. 4).

Immunohistochemically, the nuclei of the basal cells in the epithelial cell layers of the multiple non-neoplastic cysts were positive for p63 (Fig. 5a). The cytoplasm of the basal cells was positive for pan-CK and CK14, and most of the cells were positive for CK19 (Fig. 5b). The inner epithelial cells were positive for pan-CK, CK7, CK8, CK18, and CK19. The nuclei of the neoplastic cells including the cells face to the cystic lumen was diffusely positive for p63 (Fig. 6a), but negative for CK5/6, CK7, CK8, CK14, CK18, vimentin, and $\alpha$-SMA. The cytoplasm of the neoplastic cells was diffusely positive for pan-CK and CK19 (Fig. 6b), and partially positive for CK14. The innermost capsulated non-neoplastic epithelial cells were positive for pan-CK, CK7, CK8, CK18, and CK19. In both cases, no positive neoplastic cells for Syna, NSE, and Ch A were found. The intratumoral Ki-67 nuclear index was $3.7 \%$ and $2.1 \%$ in cases 1 and 2 , respectively. In the positive control tissues, the epidermis expressed pan-CK and expression of CK14 was detected in the middle to lower layer, and p63 was detected in the nuclei of the basal cell layer. Ki-67-positive nuclei were scattered throughout the basal layer. The intra sectional smooth muscle and the vascular walls of the dermal layer were positive for $\alpha$-SMA, and myoepithelial cells around the cutaneous appendages expressed $\alpha$-SMA, and CK14. In the canine liver 


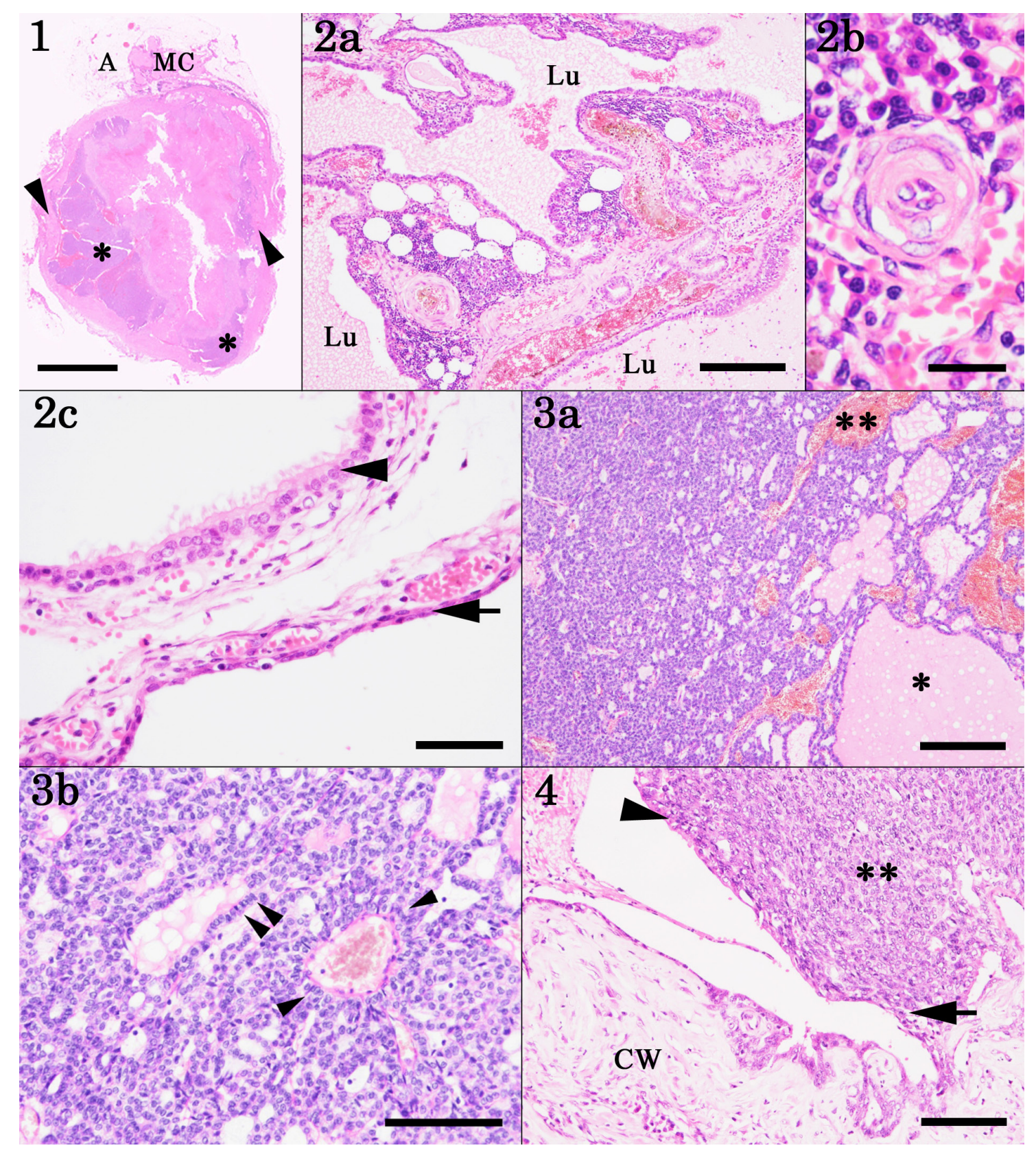

Fig. 1. Histological image of a sagittal section of the mediastinal mass in case 1. The mass is composed of multiple non-neoplastic cysts $(\mathrm{MC})$ embedded in adipose tissue (A) and proliferating neoplastic cells $\left(^{*}\right)$ in a large cyst with thick fibrous walls (arrowheads). Hematoxylin and eosin (HE). Bar $=1 \mathrm{~cm}$.

Fig. 2. Histological images of the multiple non-neoplastic cysts seen in case 1. a. The cysts wall often project into the lumen (Lu), which contained eosinophilic material, and is composed of epithelial lining cells. The cysts are surrounded by fibrous tissue of varying thickness and aggregated lymphoid tissue admixed with occasional adipose tissue. Hematoxylin and eosin (HE). Bar=500 $\mu \mathrm{m}$. b. Hassall's corpuscles are seen within the lymphoid tissue. HE. Bar $=20 \mu \mathrm{m}$. c. The cyst walls are lined by one or more layers of epithelial cells. The innermost epithelial cells are ciliated and cuboidal (arrowhead) or unciliated and squamous (arrow) in morphology. HE. Bar=50 $\mu \mathrm{m}$.

Fig. 3. Histological images of the mediastinal mass in case 2. a. The stratified neoplastic cells proliferate diffusely and are accompanied with cystic spaces filled with eosinophilic material (*) or red blood cells $(* *)$. Hematoxylin and eosin (HE). Bar=200 $\mu$ m. b. The nuclei of the neoplastic cells exhibit palisading along the interface of the cystic space and vascular structures (arrowheads). HE. Bar=100 $\mu \mathrm{m}$.

Fig. 4. Histological images of the mediastinal mass in case 1 . The regions of proliferating neoplastic cells (**) extend contiguously from the basal cells of the cyst wall epithelium (arrow) to the intracystic cavity, and the surface of the neoplastic tissue is covered by the lining of the innermost epithelium of the cyst wall (arrowhead). CW: cyst wall, Hematoxylin and eosin (HE). Bar=100 $\mu \mathrm{m}$. 


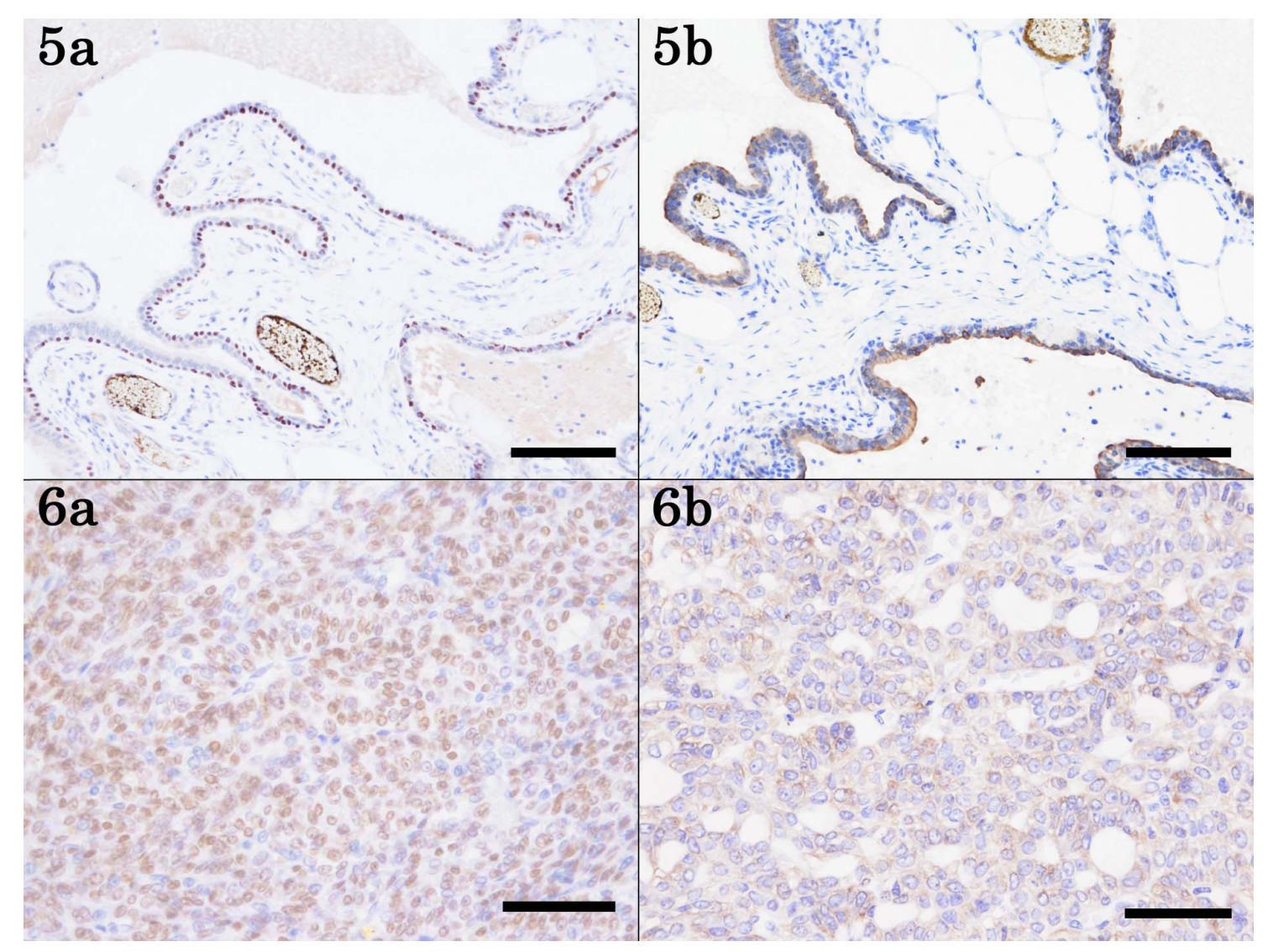

Fig. 5. Immunohistochemical examinations of the mediastinal mass in case 2. a. The nuclei in the basal layer of non-neoplastic cyst express p63. Immunostaining of p63 counterstained with hematoxylin. Bar=100 $\mu \mathrm{m}$. b. The inner cells and most of the basal cell layer of non-neoplastic cyst express cytokeratin (CK) 19. Immunostaining of CK19 counterstained with hematoxylin. Bar=100 $\mu \mathrm{m}$.

Fig. 6. Immunohistochemical examination of the mediastinal mass in case 2. a. The nuclei of the neoplastic cells express p63. Immunostaining of p63 counterstained with hematoxylin. Bar $=50 \mu \mathrm{m}$. b. The neoplastic cells express CK19. Immunostaining of CK19 counterstained with hematoxylin. Bar $=50 \mu \mathrm{m}$.

tissue, CK7, CK8, CK18 and CK19 were detected in the bile ducts, and the hepatocytes were positive for CK8 and CK18. In the adrenal gland, positive expression of Syna, NSE, and Ch A was detected in the medullary cells.

Based on these histological and immunohistochemical findings, the mediastinal masses were diagnosed as basaloid carcinomas that arose from multiple thymic cystic lesions. In animals, there have only been two reports about basaloid carcinoma. One report was about BSCC occurring in the canine lung [10], and the other report was about a pulmonary tumor in a dolphin, which was diagnosed as a poorly differentiated squamous cell carcinoma, but its histological features suggested that it might have been a BSCC [4]. The occurrence of basaloid carcinoma in animals is rare, and to the best of our knowledge, this is the first report about basaloid carcinoma in the mediastinal cavity in animals (two dogs).

In human, basaloid carcinoma is classified as a thymic tumor, and p63 is diffusely expressed in the proliferating neoplastic cells [12]. Basaloid carcinoma with squamous epithelial differentiation is classified as a type of BSCC [2], and the differentiation of basaloid carcinoma into neuroendocrine cells [9] and myoepithelial cells [5] has also been reported. In the current cases, the proliferating neoplastic cells diffusely expressed p63 and CK19, and partially expressed CK14, but none of the neoplastic cells expressed CK5/6, $\alpha$-SMA, Syna, NSE, and Ch A. These results indicated that the proliferating neoplastic basaloid cells had not differentiated into squamous epithelial, neuroendocrine, or myoepithelial cells.

The lesions in the two cases were equivalent to "thymic cysts" based on their sites of occurrence and histomorphological characteristics [15]. The thymus develops from the third and fourth pharyngeal sacs, which are covered by the epithelium of the fourth branchial arch, and gradually invades and descends as it grows, resulting in organ formation [11]. During the development of the thymus, thymic cysts can occur due to congenital abnormalities derived from the remnants of the fourth branchial arch epithelium [11]. The finding of a ciliated epithelium in the lining epithelia of thymic cysts during histological examinations is a characteristic feature that is indicative of the presence of remnants of the branchial epithelium. Cystic lesions that are histomorphologically similar to those seen in the current cases are also called "thymic branchial cysts" and have been reported in animals, including dogs $[3,8]$. 
Thymic carcinoma is defined as a tumor that is derived from the thymic epithelium [14]. Among the human mediastinal epithelial tumors originating from the thymus, basaloid carcinoma is classified as a type of thymic carcinoma [12]. This tumor is frequently accompanied by multiple cystic lesions, which are often reported to be associated with the development and/or origin of the tumor $[2,6,14]$. The cystic lesions is usually covered with an unciliated epithelial cell lining, which are assumed to originate from the thymic epithelium. In the present two cases, the histological characteristics of the cystic epithelial cell linings suggested that the cysts had originated from the remnants of the branchial arch epithelium, and the neoplastic lesions had arisen from the cysts. Therefore, based on the definition of thymic carcinoma and the origins of the neoplastic cells, the most appropriate diagnosis was considered to be basaloid carcinoma arising from thymic cysts rather than "thymic" basaloid carcinoma.

In veterinary medicine, there have been two case reports about canine mediastinal malignant tumors in the English literature, which were associated with thymic cysts (thymic branchial cysts) [1,7]. The origins of the neoplastic cells were not examined using immunohistochemical techniques in either of these cases, and the presence of nests of proliferating neoplastic cells led to the lesions being histologically diagnosed as carcinomas. In one of the cases, the neoplastic cells had invaded the surrounding stromal tissue, and metastasis to the lung was seen [7], and in the other case the infiltration of the tumor cells into the underlying stroma was observed [1]. Although mild invasion of the cyst wall by neoplastic basaloid cells was detected in the current cases, tumor proliferation was only seen in the intracystic cavity, and no metastatic lesions were found in other organs. In addition, the neoplastic cells exhibited slight atypia and low proliferative activity. Therefore, the two cases of mediastinal basaloid carcinoma were classified as being of low malignancy, according to their histological and clinical findings.

This report describes cases of basaloid carcinoma arising from thymic cyst walls in two dogs. The histopathological findings of these cases indicated that the mediastinal tumors originated from cysts derived from residual epithelial tissue left behind after thymic development. Specifically, it was concluded that the tumors originated from the basal cells of the cyst walls. Two similar canine cases have been reported previously $[1,7]$; however, the current report adds extra information about differential diagnosis among tumors that occur in the canine mediastinal cavity. Furthermore, comparative characteristic features of CT images of lymphoma and thymic epithelial tumor, which the most commonly occur in the canine mediastinal cavity, have been reported recently [13]. The current report described a mediastinal tumor that histologically different from lymphoma and thymoma, therefore, the clinical information in the current report also provides information to clinical veterinarians about other type of neoplastic lesion in the mediastinum of dogs. Although the two present cases had rare origins, basaloid carcinoma should be included in the list of differential diagnoses for canine mediastinal tumors.

POTENTIAL CONFLICTS OF INTEREST. The authors declare that there are no potential conflicts of interest with this manuscript.

\section{REFERENCES}

1. Adam, R., McAlinden, A. B., Llabrés-Díaz, F. J. and Lelyveld, S. 2014. Thymic branchial cyst carcinoma in a dog. Research 1: 645-649.

2. Brown, J. G., Familiari, U., Papotti, M. and Rosai, J. 2009. Thymic basaloid carcinoma: a clinicopathologic study of 12 cases, with a general discussion of basaloid carcinoma and its relationship with adenoid cystic carcinoma. Am. J. Surg. Pathol. 33: 1113-1124. [Medline] [CrossRef]

3. Day, M. J. 1997. Review of thymic pathology in 30 cats and 36 dogs. J. Small Anim. Pract. 38: 393-403. [Medline] [CrossRef]

4. Ewing, R. Y. and Mignucci-Giannoni, A. A. 2003. A poorly differentiated pulmonary squamous cell carcinoma in a free-ranging Atlantic bottlenose dolphin (Tursiops truncatus). J. Vet. Diagn. Invest. 15: 162-165. [Medline] [CrossRef]

5. Hishida, T., Nakanishi, Y., Shimoda, T., Igaki, H., Tachimori, Y., Kato, H., Yamaguchi, H. and Iinuma, G. 2002. Esophageal basaloid carcinoma with marked myoepithelial differentiation. Pathol. Int. 52:313-317. [Medline] [CrossRef]

6. Iezzoni, J. C. and Nass, L. B. 1996. Thymic basaloid carcinoma: a case report and review of the literature. Mod. Pathol. 9: 21-25. [Medline]

7. Levien, A. S., Summers, B. A., Szladovits, B., Benigni, L. and Baines, S. J. 2010. Transformation of a thymic branchial cyst to a carcinoma with pulmonary metastasis in a dog. J. Small Anim. Pract. 51: 604-608. [Medline] [CrossRef]

8. Liu, S., Patnaik, A. K. and Burk, R. L. 1983. Thymic branchial cysts in the dog and cat. J. Am. Vet. Med. Assoc. 182: 1095-1098. [Medline]

9. Majhi, U., Murhekar, K., Sundersingh, S. and Srinivasan, V. 2015. Basaloid squamous cell carcinoma of cervix showing neuroendocrine differentiation. J. Cancer Res. Ther. 11: 492-493. [Medline] [CrossRef]

10. Morey-Matamalas, A., de Stefani, A., Corbetta, D., Grau-Roma, L. and de Brot, S. 2018. Pulmonary basaloid squamous cell carcinoma in a dog. J. Comp. Pathol. 159: 11-15. [Medline] [CrossRef]

11. Nelson, L. L., Coelho, J. C., Mietelka, K. and Langohr, I. M. 2012. Pharyngeal pouch and cleft remnants in the dog and cat: a case series and review. J. Am. Anim. Hosp. Assoc. 48: 105-112. [Medline] [CrossRef]

12. Papotti, M., Ströbel, P., Marx, A., Chen, J. K. C. and Detterbeck, F. 2015. Basaloid carcinoma. pp. 216-217. In: WHO classification of Tumors of the Lung, Pleura, Thymus and Heart, 4th ed. (Travis, W. D., Brambilla, E., Burke, A. P., Marx, A. and Nicholson, A. G., eds.), International Agency for Research on Cancer, Lyon.

13. Reeve, E. J., Mapletoft, E. K., Schiborra, F., Maddox, T. W., Lamb, C. R. and Warren-Smith, C. M. R. 2020. Mediastinal lymphoma in dogs is homogeneous compared to thymic epithelial neoplasia and is more likely to envelop the cranial vena cava in CT images. Vet. Radiol. Ultrasound 61: 25-32. [Medline] [CrossRef]

14. Valli, V. E., Bienzle, D., Meuten, D. J. and Linder, K. E. 2017. Tumors of the hemolymphatic system. pp. 203-321. In: Tumors in domestic animals, 5th ed. (Meuten, D. J., ed.), Wiley Blackwell, Ames.

15. Valli, V. E., Kiupel, M. and Bienzle, D. (with Wood, R. D.). 2015. Hematopoietic system. pp. 102-268. In: Jubb, Kennedy, Palmer's Pathology of Domestic Animals, Vol.3, 6th ed. (Maxie, M. G., ed.), Elsevier, St. Louis. 